\title{
European survey on the use of prophylactic fluconazole in neonatal intensive care units
}

\author{
Florentia Kaguelidou • Chiara Pandolfini • \\ Paolo Manzoni • Imti Choonara • Maurizio Bonati • \\ Evelyne Jacqz-Aigrain
}

Received: 6 July 2011 / Accepted: 31 August 2011 / Published online: 14 September 2011

(C) The Author(s) 2011. This article is published with open access at Springerlink.com

\begin{abstract}
Neonatal fungal infections are associated with substantial mortality and morbidity. Although prophylactic use of several antifungals has been proposed, this practice remains controversial. In order to evaluate the use of fluconazole prophylaxis in European NICUs, we conducted a cross-sectional survey by means of a structured questionnaire that was sent to European level II and III neonatal intensive care units, over a 9-month period, as part of a neonatal research FP7 European project. A total of 193 questionnaires from 28 countries were analysed. Use of antifungal prophylaxis was reported by $55 \%$ of the respond-
\end{abstract}

F. Kaguelidou $\cdot$ E. Jacqz-Aigrain

Paediatric Pharmacology and Pharmacogenetics Department,

APHP, Hôpital Robert Debré; INSERM CIC9202,

Université Paris 7 ,

Paris, France

C. Pandolfini $\cdot$ M. Bonati

Laboratory for Mother and Child Health, Department of Public

Health, 'Mario Negri' Pharmacological Research Institute,

Milan, Italy

P. Manzoni

Neonatology and Hospital Neonatal Intensive Care Unit,

Azienda Ospedaliera Regina Margherita-S Anna,

Torino, Italy

I. Choonara

Academic Division of Child Health, University of Nottingham,

Derbyshire Children's Hospital,

Derby, UK

E. Jacqz-Aigrain $(\bowtie)$

Department of Paediatric Pharmacology and Pharmacogenetics; Clinical Investigation Center, CIC; Inserm 9202,

Hôpital Robert Debré,

48 boulevard Sérurier 75019 ,

Paris, France

e-mail: evelyne.jacqzaigrain@gmail.com ers, and the most frequently used antifungal agent was fluconazole $(92 \%)$. Main indications for prophylaxis were low gestational age $(<28$ weeks) and birth weight $(<1,000 \mathrm{~g})$. A dose of $3 \mathrm{mg} / \mathrm{kg}$ was used in $66 \%$ of NICUs using fluconazole, with an administration interval of $72 \mathrm{~h}$ in $52 \%$ of them. All responders acknowledged the need for additional trials on the efficacy of prophylactic fluconazole. Non-users of fluconazole prophylaxis were more likely to be influenced by the local incidence of candidiasis, the risk of increasing antifungal resistance and the absence of specific recommendations by paediatric societies. Conclusions: Major concerns about the use of fluconazole prophylaxis include its efficacy, the risk of emergence of resistant species and the absence of clear consensus to support routine use. Future studies that address these issues will contribute to a more rational use of fluconazole prophylaxis.

Keywords Fluconazole · Prophylaxis · Neonatology · Intensive care $\cdot$ Survey

$\begin{array}{ll}\text { Abbreviations } \\ \text { IFIs } & \text { Invasive fungal infections } \\ \text { VLBW } & \text { Very low birth weight } \\ \text { ELBW } & \text { Extremely low birth weight infants } \\ \text { NICUs } & \text { Neonatal intensive care units } \\ \text { RCT } & \text { Randomized controlled trial } \\ \text { GA } & \text { Gestational age } \\ \text { TINN } & \text { Treat infections in neonates }\end{array}$

\section{Introduction}

Neonatal fungal infections, mainly due to Candida spp., are often associated with substantial mortality and morbidity. 
Major risk factors include low gestational age and very low birth weight (VLBW, $<1,500 \mathrm{~g}$ ), use of steroids and broad-spectrum antibiotics, prolonged presence of a central venous catheter and use of parenteral nutrition [13]. Estimated incidence of invasive fungal infections (IFIs) is 2\% in VLBW neonates and 3\% to more than $23 \%$ in extremely low birth weight (ELBW, $<1,000 \mathrm{~g}$ ) neonates. Also, Candida-associated mortality rates are high, especially in ELBW infants, $73 \%$ of which either do not survive or survive with neurodevelopmental impairment [3]. In addition, the real burden of neonatal IFIs is probably underestimated because of diagnostic limitations due to their subtle clinical presentation, frequent laboratory difficulties in identifying the pathogen and absence of a consensus definition for IFIs [14].

Given the severity of these infections, the prophylactic use of several antifungal agents has been proposed. Successful results concerning the azole agent fluconazole have prompted certain neonatal intensive care units (NICUs) to adopt its use in prophylaxis mainly in the population of extremely premature infants. Yet, this medical practice remains controversial [20]. In a US survey conducted in 2004 , only $34 \%$ of the participating neonatologists reported the use of antifungal prophylaxis [5]. From that survey to date, eight retrospective studies $[1,4$, $10,11,15,22,26,29]$, two prospective randomized controlled trials (RCTs) $[16,21]$ and a Cochrane metaanalysis [7] have demonstrated the efficacy of fluconazole prophylaxis. Hence, the Infectious Disease Society of America 2009 guidelines recommend fluconazole prophylaxis "for premature infants and infants with ELBW in nurseries that have a high incidence of invasive candidiasis" [23]. In this context, we aimed to evaluate the use of fluconazole for the prevention of IFIs and the factors that influence this practice in European NICUs.

\section{Materials and methods}

\section{Selection of study participants}

This survey was part of a wider study conducted by the FP7 European project Treat Infections in Neonates (TINN) research network. This network was established by the European Commission in 2007 to evaluate the use of two off-patent medicines in neonates: ciprofloxacin and fluconazole [9]. This collaborative project has brought together a group of neonatal health experts from seven countries. To conduct the survey, given the absence of an exhaustive list of European NICUs, the TINN experts were asked to identify units located in their respective countries and in other European countries. Also, paediatric European networks and national societies were contacted to provide additional information on existing NICUs. Between December 2009 and May 2010, a first invitation to participate in the online survey was emailed to the heads of all identified NICUs. Then, a second email was sent in June 2010 to urge clinicians to either participate or complete provided information. The invitation to participate mentioned that the study involved the use of an antimicrobial agent without specifying the drug. Also, neonatologists initially contacted were urged to pass the invitation on to other heads of NICUs.

As the characteristics of NICUs differ widely in Europe [27], we analysed data provided by level II and III neonatal care units as defined in a previous publication [25]. Also, only one completed questionnaire per unit was analysed.

\section{Survey}

The survey was conducted by means of a questionnaire that was directly completed by neonatologists online. This questionnaire had been constructed and pre-tested by TINN experts. The following information were assessed: characteristics of the unit (level of care, total number of neonatal beds, average annual admissions of neonates and those of newborns less than 32 weeks' gestational age (GA), estimated monthly rate of sepsis, prevalence of fungal infections) and use or not of fluconazole prophylaxis. We considered a prevalence of fungal infections of less than $1 \%$ to be low, based on previous published data $[13,23]$. For units prescribing prophylactic fluconazole, clinical indications, drug doses and dosing schedules, reasons for discontinuation and the nature of the agent prescribed for treatment of confirmed fungal infection were noted. Information on the following items - number of NICU beds, average annual admission of neonates, estimated prevalence of fungal infections, use or not of prophylactic fluconazole-were compulsory for inclusion in the survey analysis.

Each unit's rationale regarding fluconazole prophylaxis was evaluated by the responses given to nine items addressing different factors that may have influenced this practice. Responses were measured on a 5-point Likert scale (1, least important; 5, most important) and were further dichotomized: responses $\leq 3$ were considered as less important and those $\geq 4$ as very important. We integrated the same items as in the 2004 US survey [5].

Statistical analysis

Descriptive data included absolute numbers (percentages) for categorical variables and medians [1st-3rd quartile] for continuous variables. To compare proportions, we used a chi-square or Fisher's exact test and the Wilcoxon nonparametric test to compare medians. All differences were 
significant at a $p<0.05$ level. Statistical analyses were performed using SAS version 9.2 software.

\section{Results}

By September 2010, 327 neonatologists had accessed the online survey, and 226 had inputted any data. Further, 33 questionnaires were excluded because they were duplicates $(n=22)$, compulsory information was missing $(n=4)$ and they were provided by non-European countries $(n=5)$ or by level I NICUS $(n=2)$. Thus, a total of 193 questionnaires from 193 units located in 28 countries were analysed; those with the greatest number of NICUs that joined the survey were Italy, the UK and France.

\section{Characteristics of participating NICUs}

Most NICUS were level III units (167 of $193,86.5 \%$ ) with a median number of neonatal beds of $14(8-22)$ and a mean of more than 120 admissions of newborns (160 of 193, $83 \%$ ) per year. The majority reported annual admissions of preterm neonates of either 50 to 100 (77 of $193,40 \%)$ or 100 to 200 (58 of 193, 30\%). In $72 \%$ (139 of 193) of the units, the monthly rate of bacterial sepsis was estimated between $1 \%$ and $10 \%$. The reported prevalence of fungal infections was less than $1 \%$ in 125 units $(65 \%), 1 \%$ to $5 \%$ in $56(29 \%)$ and $5 \%$ to $15 \%$ in $12(6 \%)$ NICUs.

\section{Use of fluconazole prophylaxis}

One hundred and seven NICUs (55\%) reported use of antifungal prophylaxis. Of those, $75 \%$ (80 of 107) stated the presence of a written protocol regarding routine antifungal prophylaxis against only $26 \%$ (22 of 86 ) of the units which did not use prophylaxis $(p<0.0001)$. There were no other statistically significant differences concerning the unit characteristics described above. In particular, no difference was found in the prevalence of fungal infections between users and non-users of antifungal prophylaxis (prevalence $<1 \%, 65$ of $107(61 \%)$ and 60 of $86(70 \%)$, respectively; $p=0.19)$.

Fluconazole was the most frequently used prophylactic agent (99 of $107,92 \%)$. Nystatin or miconazole $(n=3$; agents not stated, $n=5$ ) was also prescribed. Details on the indications to initiate fluconazole prophylaxis are given in Table 1. Main indications for prophylaxis were a low GA and weight at birth, and the most commonly reported cutoffs were GA less than 28 weeks and birth weight less than $1,000 \mathrm{~g}$. Almost half of the units initiate prophylaxis upon positive fungal colonization; however, routine surveillance cultures are performed by $58 \%$ (29 of 50 ) of these units, and the remaining determine colonization status on cultures motivated by clinical features ( 21 of $50,42 \%$ ).
Fluconazole was more often given intravenously (81\%) than orally (19\%). A mean dose of $3 \mathrm{mg} / \mathrm{kg}$ was used in $66 \%$ of NICUs $(6 \mathrm{mg} / \mathrm{kg}, 23 \%$; other, $6 \%$; missing, $5 \%$ ), with an administration interval of $72 \mathrm{~h}$ in $52 \%$ of them (48 h, 22\%; $24 \mathrm{~h}, 10 \%$; other, 11\%; missing, 5\%). Antifungal prophylaxis was discontinued after a predetermined duration (range, 10 to 45 days) in $38 \%$ of the units, when the intravenous route was no longer available in $35 \%$ and when an appropriate corrected GA (range, 2833 weeks) or target weight (range, 1,000 to $2,000 \mathrm{~g}$ ) was reached in $9 \%$ and $6 \%$ of NICUS, respectively. Finally, $57 \%$ of the NICUs that use fluconazole prophylaxis reported the administration of amphotericin B for the treatment of a confirmed fungal infection. In the same clinical context, fluconazole was prescribed in $23 \%$ of these NICUs, flucytozine and caspofungin in $5 \%$ each and micafungin in $1 \%$ (missing data, $9 \%$ ).

\section{Rationale for fluconazole prophylaxis}

Overall, the items that were considered as most influential on the decision to use or not to use fluconazole prophylaxis were the NICU's incidence of candidiasis, the risk of increasing drug resistance related to its widespread use and the need for additional efficacy studies. Details are given in Table 2 .

However, differences were observed between NICUs that used prophylactic fluconazole and those that did not use prophylaxis. Neonatologists who did not prescribe routine prophylaxis were more likely to be influenced by the incidence of candidiasis in their unit, the risk of increasing antifungal resistance and the absence of a statement by paediatric societies in support of routine prophylaxis use than those who used prophylactic fluconazole (Table 2). Finally, neonatologists that did not prescribe prophylaxis were more concerned about the need for validated criteria to define high-risk neonates, including surveillance cultures, although differences did not reach significance.

\section{Discussion}

This is the first survey on the practices regarding antifungal prophylaxis among level II and III NICUs from 28 European countries. Approximately half of the participating neonatologists reported regular use of fluconazole prophylaxis in their NICUs. This survey gives evidence on how widely prophylaxis administration modalities vary across European NICUs and highlights three key factors influencing the prophylaxis decision process: the local incidence of candidiasis, the potential risk of increasing antifungal resistance and the need for additional efficacy trials. 
Table 1 Indications for use of prophylactic fluconazole

\begin{tabular}{|c|c|c|c|c|}
\hline \multirow[t]{2}{*}{ Indications for prophylaxis } & & \multicolumn{2}{|c|}{ NICUs using fluconazole prophylaxis $(n=99)$} & \multirow[t]{2}{*}{ Number of available data } \\
\hline & & $n$ & $\%$ & \\
\hline \multirow[t]{5}{*}{ Birth gestational age } & No & 25 & 27 & 93 \\
\hline & $<26$ weeks & 21 & 23 & \\
\hline & $<28$ weeks & 30 & 32 & \\
\hline & $<30$ weeks & 15 & 16 & \\
\hline & $<32$ weeks & 2 & 2 & \\
\hline \multirow[t]{5}{*}{ Birth weight } & No & 19 & 20 & 93 \\
\hline & $<750 \mathrm{~g}$ & 14 & 15 & \\
\hline & $<1,000 \mathrm{~g}$ & 37 & 40 & \\
\hline & $<1,500 \mathrm{~g}$ & 22 & 24 & \\
\hline & Other $(1,250 \mathrm{~g})$ & 1 & 1 & \\
\hline \multirow[t]{4}{*}{ Neonate receiving antibiotics for: } & No & 38 & 41 & 92 \\
\hline & $>2$ days & 12 & 13 & \\
\hline & $>7$ days & 25 & 27 & \\
\hline & $>14$ days & 17 & 19 & \\
\hline \multirow[t]{2}{*}{ Presence of central venous catheter } & Yes & 51 & 55 & 92 \\
\hline & No & 42 & 45 & \\
\hline \multirow[t]{2}{*}{ Positive colonization status } & Yes & 50 & 52 & 96 \\
\hline & No & 46 & 48 & \\
\hline \multirow[t]{2}{*}{ Presence of endotracheal intubation } & Yes & 23 & 25 & 92 \\
\hline & No & 69 & 75 & \\
\hline \multirow[t]{2}{*}{ Total parental nutrition in use } & Yes & 35 & 38 & 92 \\
\hline & No & 57 & 62 & \\
\hline \multirow[t]{2}{*}{ Abdominal surgery } & Yes & 23 & 26 & 90 \\
\hline & No & 67 & 74 & \\
\hline \multirow[t]{2}{*}{ Abdominal disease } & Yes & 22 & 24 & 90 \\
\hline & No & 68 & 76 & \\
\hline
\end{tabular}

Table 2 Influence of nine factors in the decision whether or not to prescribe prophylactic fluconazole

\begin{tabular}{|c|c|c|c|c|c|c|}
\hline \multirow[t]{2}{*}{ Rationale for practice } & \multicolumn{2}{|c|}{$\begin{array}{l}\text { Use fluconazole } \\
\text { prophylaxis } \\
(n=99), n(\%)\end{array}$} & \multicolumn{2}{|c|}{$\begin{array}{l}\text { Do not use prophylaxis } \\
(n=86), n(\%)\end{array}$} & \multirow[t]{2}{*}{$\begin{array}{l}\text { Number of } \\
\text { available data }\end{array}$} & \multirow[t]{2}{*}{$p$ values } \\
\hline & Likert $\leq 3$ & Likert $\geq 4$ & Likert $\leq 3$ & Likert $\geq 4$ & & \\
\hline $\begin{array}{l}\text { Incidence of candidiasis in your NICU is/is not } \\
\text { high enough to justify prophylaxis }\end{array}$ & $55(59)$ & $39(41)$ & $17(21)$ & $64(79)$ & 181 & $<0.0001$ \\
\hline $\begin{array}{l}\text { Widespread antifungal use could lead to increased } \\
\text { antifungal resistance }\end{array}$ & $48(51)$ & $46(49)$ & $25(32)$ & $54(68)$ & 178 & 0.01 \\
\hline $\begin{array}{l}\text { Statement by paediatric societies in support of } \\
\text { routine use in a subset of newborns is needed }\end{array}$ & $53(60)$ & $36(40)$ & $35(44)$ & $44(56)$ & 174 & 0.048 \\
\hline $\begin{array}{l}\text { The criteria of high-risk patients in whom prophylaxis } \\
\text { should be attempted need clarification }\end{array}$ & $40(60)$ & $27(40)$ & $37(46)$ & $44(54)$ & 179 & 0.23 \\
\hline The agent is too costly & $89(96)$ & $4(4)$ & $74(94)$ & $5(6)$ & 178 & 0.73 \\
\hline $\begin{array}{l}\text { The role of surveillance culture in identifying } \\
\text { high-risk neonates needs clarification }\end{array}$ & $50(55)$ & $41(45)$ & $39(48)$ & $42(52)$ & 177 & 0.37 \\
\hline $\begin{array}{l}\text { Uncertainty about pharmacometrics of the antifungal } \\
\text { agent in the newborn is great }\end{array}$ & $62(67)$ & $31(33)$ & $48(60)$ & $32(40)$ & 178 & 0.36 \\
\hline $\begin{array}{l}\text { Uncertainty about safety of the antifungal agent } \\
\text { in the newborn is great }\end{array}$ & 67 (73) & $25(27)$ & $53(65)$ & $28(35)$ & 178 & 0.29 \\
\hline $\begin{array}{l}\text { Additional studies of efficacy of the antifungal agent } \\
\text { in the perinatal population are needed }\end{array}$ & $41(43)$ & $54(57)$ & 29 (36) & $52(64)$ & 181 & 0.32 \\
\hline
\end{tabular}


Fluconazole is the most evaluated agent for the prevention of IFIs in preterm neonates [7, 18]. In our survey, we noticed variations among NICUs in the dosing schedule, the frequency of administration and the duration of fluconazole prophylaxis. Although usually administered intravenously, several units still administer fluconazole or other antifungal agents orally. Fluconazole has a good oral absorption, but the action of other antifungal agents like oral nystatin or miconazole is limited to the gastroenteric tract, and their use is not supported by evidence from highlevel RCTs $[2,16]$. Such diversity of practices with regard to antifungal prophylaxis is in accordance with data from previous surveys $[5,8]$ and highlights the need to establish recommendations for fluconazole administration.

Furthermore, most participating neonatologists agreed upon the need for additional efficacy data on prophylactic fluconazole, as expressed in the 2004 US survey [5]. Nevertheless, the use of fluconazole is supported by highlevel evidence including five well-conducted RCTs that enrolled a total of 656 neonates [6, 15, 17, 21, 24]. Metaanalysis of these RCTs demonstrated that prophylactic fluconazole significantly reduces the incidence of IFIs in VLBW infants when compared to a placebo, with a number of neonates needed to treat of 11 [7]. If neonatologists still judge efficacy data as insufficient, then what is missing in medical literature? First, no significant reduction on mortality by prophylactic fluconazole has been demonstrated. Long-term outcomes, such as disability-free survival, have not been published. Both mortality and neurodevelopmental assessment may represent more adequate trial endpoints to evaluate prophylactic medication because they are direct consequences of IFIs and they are not influenced by ascertainment bias as opposed to the incidence of IFIs. However, these two outcomes in preterm infants are multifactorial; thus, designing appropriate trials to assess them is challenging. Second, as argued by non-users of prophylaxis, neonates at high risk of IFIs and the role of surveillance cultures need to be defined. Although most clinicians agree to target the youngest and smallest newborns, age and weight cutoffs to initiate prophylaxis differ widely, and practices are even more heterogeneous for other risk factors such as antibiotic use and fungal colonization status. All published RCTs were underpowered to perform subgroup analysis, and the role of fungal colonization in the progression of the disease, although suggested [19], has never been prospectively evaluated. This may partly explain why paediatric societies have not issued recommendations on antifungal prophylaxis in a targeted subset of neonates.

Also, most participating neonatologists agreed that uncertainty about the pharmacometrics or safety of prophylactic fluconazole does not influence their practices. Yet, neonatal pharmacokinetic data of fluconazole to support adequate dosing are limited and are totally missing in neonates less than $750 \mathrm{~g}$ [28]. Likewise, prospective longterm safety data, focusing on potential neurodevelopmental impact, are missing in premature neonates.

The prevalence of fungal infections varies widely between units because of differences in medical practices like the management of central venous catheters or the use of broad-spectrum antibiotics. In units where the rate of infections is low $(<1 \%)$, prophylactic fluconazole is not necessary. However, a difference in infection rates between NICUs using and those not using prophylaxis was not evidenced in our survey. Also, the real incidence of IFIs in a NICU remains difficult to determine because of the frequent inability to yield the causal pathogen from cultures in neonates and of the absence of a standardized definition for IFIs $[6,13]$.

Moreover, clinicians who do not support antifungal prophylaxis are more concerned about the potential risk of increasing resistance as a consequence of its widespread use. To date, several studies have addressed this issue, and none has reported the emergence of fluconazole-resistant Candida species [12, 15, 21]. Nevertheless, long-term mycological surveillance data are currently not available. Overall, the findings of our survey are similar to those of the US survey [5], where neonatologists who did not use fluconazole prophylaxis were also more concerned about the emergence of resistance and the need to further define high-risk neonates and evaluate the role of surveillance cultures. However, information on the impact of the local prevalence of fungal infections was not reported in that article.

Due to the absence of an exhaustive list of NICUs and the wide circulation of the survey's invitation, the total number of units invited to participate is imprecise, and a global response rate cannot be estimated. The above reasons could also account for non-participation (survey accessed online but questionnaire not completed) as part of the contacted clinicians might not provide medical care for very preterm and low birth weight neonates. In addition, the number of questionnaires available from the different $\mathrm{EU}$ countries varies, with Italy, the UK and France being predominantly represented. This did not allow precise geographic analysis of differences in fluconazole prophylactic use between Northern and Southern Europe. Nevertheless, the main objective of the survey was not to quantify the frequency of use of fluconazole prophylaxis and to extrapolate it at a global European level. Finally, we did not question all practising neonatologists in each NICU, and we did not test the respondents' knowledge of the current literature. However, questionnaires were completed by the heads of NICUs, whose opinion is frequently reflective of the practices in a unit and whose status may be a proxy for literature knowledge. 


\section{Conclusion}

Use and dosage schedule of routine fluconazole prophylaxis are currently variable among European NICUs. Responders acknowledged the need for additional trials to evaluate the efficacy of prophylactic fluconazole regardless of their respective practice of prophylaxis. Nevertheless, neonatologists who did not use fluconazole prophylaxis were more likely to be influenced by the local incidence of candidiasis, the risk of emergence of antifungal resistances and the absence of a statement by paediatric societies to support routine use of prophylaxis. Future studies that will address these issues will contribute to a more rational and safer use of antifungal fluconazole prophylaxis.

Acknowledgements We would like to thank all neonatologists that participated in this survey. Also, we thank Pr. Corinne Alberti and all the other TINN partners for helpful discussions.

Funding statement This work is part of the TINN network (Collaborative Project) supported by the European Commission under the Health Cooperation Work Programme of the 7th Framework Programme (grant agreement no. 223614). The funders had no role in study design, data collection and analysis, decision to publish or preparation of the manuscript.

Competing interests The authors have no conflict of interest.

Open Access This article is distributed under the terms of the Creative Commons Attribution Noncommercial License which permits any noncommercial use, distribution, and reproduction in any medium, provided the original author(s) and source are credited.

\section{References}

1. Aghai ZH, Mudduluru M, Nakhla TA, Amendolla B, Lo go D, Kemble N, Kaki S, Sutsko R, Saslow JG, Stahl GE (2006) Fluconazole prophylaxis in extremely low birth weight infants: association with cholestasis. J Perinatol 26:550-555

2. Austin N, Darlow BA, McGuire W (2009) Prophylactic oral/ topical non-absorbed antifungal agents to prevent invasive fungal infection in very low birth weight infants. Cochrane Database Syst Rev 7:CD003478

3. Benjamin DK Jr, Stoll BJ, Fanaroff AA, McDonald SA, Oh W, Higgins RD, Duara S, Poole K, Laptook A, Goldberg R, Institute N, National Institute of Health and Human Development Neonatal Research Network (2006) Neonatal candidiasis among extremely low birth weight infants: risk factors, mortality rates, and neurodevelopmental outcomes at 18 to 22 months. Pediatrics 117:84-92

4. Bertini G, Perugi S, Dani C, Filippi L, Pratesi S, Rubaltelli FF (2005) Fluconazole prophylaxis prevents invasive fungal infection in high-risk, very low birth weight infants. J Pediatr 147:162-165

5. Burwell LA, Kaufman D, Blakely J, Stoll BJ, Fridkin SK (2006) Antifungal prophylaxis to prevent neonatal candidiasis: a survey of perinatal physician practices. Pediatrics 118:e1019-1026
6. Cabrera C, Frank M, Carter D, Bhatia J (2002) Fluconazole prophylaxis against systemic candidiasis after colonization: a randomised double-blinded study. J Perinatol 22:604

7. Clerihew L, Austin N, McGuire W (2007) Prophylactic systemic antifungal agents to prevent mortality and morbidity in very low birth weight infants. Cochrane database of systematic reviews CD003850

8. Clerihew L, McGuire W (2008) Antifungal prophylaxis for very low birthweight infants: UK national survey. Arch Dis Child Fetal Neonatt Ed 93:F238-239

9. EU FP7 project: http//www.tin-project.org/.

10. Fridkin SK, Kaufman D, Edwards JR, Shetty S, Horan T (2006) Changing incidence of Candida bloodstream infections among NICU patients in the United States: 1995-2004. Pediatrics 117:1680-1687

11. Healy CM, Baker CJ, Zaccaria E, Campbell JR (2005) Impact of fluconazole prophylaxis on incidence and outcome of invasive candidiasis in a neonatal intensive care unit. J Pediatr 147:166171

12. Healy CM, Campbell JR, Zaccaria E, Baker CJ (2008) Fluconazole prophylaxis in extremely low birth weight neonates reduces invasive candidiasis mortality rates without emergence of fluconazoleresistant Candida species. Pediatrics 121:703-710

13. Kaufman D (2009) Neonatal candidiasis: clinical manifestations, management, and prevention strategies. J Pediatr 156:S47-S52

14. Kaufman DA (2010) Challenging issues in neonatal candidiasis. Curr Med Res Opin 26:1769-1778

15. Kaufman D, Boyle R, Hazen KC, Patrie JT, Robinson M, Donowitz LG (2001) Fluconazole prophylaxis against fungal colonization and infection in preterm infants. $\mathrm{N}$ Engl $\mathrm{J}$ Med 345:1660-1666

16. Kaufman D, Boyle R, Hazen KC, Patrie JT, Robinson M, Grossman LB (2005) Twice weekly fluconazole prophylaxis for prevention of invasive Candida infection in high-risk infants of $<1000$ grams birth weight. J Pediatr 147:172-179

17. Kicklighter SD, Springer SC, Cox T, Hulsey TC, Turner RB (2001) Fluconazole for prophylaxis against candidal rectal colonization in the very low birth weight infant. Pediatrics 107:293-298

18. Manzoni P, Arisio R, Mostert M, Leonessa M, Farina D, Latino MA, Gomirato G (2006) Prophylactic fluconazole is effective in preventing fungal colonization and fungal systemic infections in preterm neonates: a single-center, 6-year, retrospective cohort study. Pediatrics 117:e22-32

19. Manzoni P, Farina D, Leonessa M, d'Oulx EA, Galetto P, Mostert M, Miniero R, Gomirato G (2006) Risk factors for progression to invasive fungal infection in preterm neonates with fungal colonization. Pediatrics 118:2359-2364

20. Manzoni P, Mostert M, Jacqz-Aigrain E, Farina D (2009) The use of fluconazole in neonatal intensive care units. Arch Dis Child 94:983-987

21. Manzoni P, Stolfi I, Pugni L, Decembrino L, Magnini C, Vetrano G, Tridapalli E, Corona G, Giovannozzi G, Farina D, Arisio R, Merletti F, Maule M, Mosca F, Pedicino R, Stronati M, Mostert M, Gomirato G, Italian Task Force for the Study and Prevention of Neonatal Fungal Infections; Italian Society of Neonatology (2007) A multicenter, randomized trial of prophylactic fluconazole in preterm neonates. N Engl J Med 356:2483-2495

22. McCrossan BA, McHenry E, O'Neill F, Ong G, Sweet DG (2007) Selective fluconazole prophylaxis in high-risk babies to reduce invasive fungal infection. Arch Dis Child Fetal Neonatal Ed 92: F454-458

23. Pappas PG, Kauffman CA, Andes D, Benjamin DK Jr, Calandra TF, Edwards JE Jr, Filler SG, Fisher JF, Kullberg BJ, Ostrosky-Zeichner L, Reboli AC, Rex JH, Walsh TJ, Sobel JD, Infectious Diseases Society of America (2009) Clinical practice guidelines for the 
management of candidiasis: 2009 update by the Infectious Diseases Society of America. Clin Infect Dis 48:503-535

24. Parikh TB, Nanavati RN, Patankar CV, Rao S, Bisure K, Udani RH, Mehta P (2007) Fluconazole prophylaxis against fungal colonization and invasive fungal infection in very low birth weight infants. Indian Pediatr 44:830-837

25. Stark AR (2004) Levels of neonatal care. Pediatrics 114:13411347

26. Uko S, Soghier LM, Vega M, Marsh J, Reinersman GT, Herring L, Dave VA, Nafday S, Brion LP (2006) Targeted short-term fluconazole prophylaxis among very low birth weight and extremely low birth weight infants. Pediatrics 1117:1243-1252

27. Van Reempts P, Gortner L, Milligan D, Cuttini M, Petrou S, Agostini R, Field D, den Ouden L, Borch K, Mazela J, Carrapato
M, Zeittin J, MOSAIC Research Group (2007) Characteristics of neonatal units that care for very preterm infants in Europe: results from the MOSAIC study. Pediatrics 120:e815-825

28. Wade KC, Wu D, Kaufman DA, Ward RM, Benjamin DK Jr, Sullivan JE, Ramey N, Javaraman B, Hoppu K, Adamson PC, Gastronguay MR, Barrett JS, National Institute of Child Health and Development Paediatric Pharmacology Research Network (2008) Population pharmacokinetics of fluconazole in young infants. Antimicrob Agents Chemother 52:40434049

29. Weitkamp JH, Ozdas A, LaFleur B, Potts AL (2008) Fluconazole prophylaxis for prevention of invasive fungal infections in targeted highest risk preterm infants limits drug exposure. J Perinatol 28:405-411 